\title{
Cosmic shear bias and calibration in dark energy studies
}

\author{
A. N. Taylor ${ }^{1 \star}$ and T. D. Kitching ${ }^{2}$ \\ ${ }^{1}$ Scottish Universities Physics Alliance, Institute for Astronomy, School of Physics and Astronomy, University of Edinburgh, Royal Observatory, Blackford \\ Hill, Edinburgh EH9 3HJ, UK \\ ${ }^{2}$ Mullard Space Science Laboratory, University College London, Holmbury St. Mary, Dorking, Surrey RH5 6NT, UK
}

Accepted 2018 February 9. Received 2018 February 9; in original form 2016 June 26

\begin{abstract}
With the advent of large-scale weak lensing surveys there is a need to understand how realistic, scale-dependent systematics bias cosmic shear and dark energy measurements, and how they can be removed. Here, we show how spatially varying image distortions are convolved with the shear field, mixing convergence $\mathrm{E}$ and $\mathrm{B}$ modes, and bias the observed shear power spectrum. In practise, many of these biases can be removed by calibration to data or simulations. The uncertainty in this calibration is marginalized over, and we calculate how this propagates into parameter estimation and degrades the dark energy Figure-of-Merit. We find that noise-like biases affect dark energy measurements the most, while spikes in the bias power have the least impact. We argue that, in order to remove systematic biases in cosmic shear surveys and maintain statistical power, effort should be put into improving the accuracy of the bias calibration rather than minimizing the size of the bias. In general, this appears to be a weaker condition for bias removal. We also investigate how to minimize the size of the calibration set for a fixed reduction in the Figure-of-Merit. Our results can be used to correctly model the effect of biases and calibration on a cosmic shear survey, assess their impact on the measurement of modified gravity and dark energy models, and to optimize survey and calibration requirements.
\end{abstract}

Key words: methods: data analysis-methods: statistical-cosmological parameters - large-scale structure of Universe.

\section{INTRODUCTION}

The observed accelerated expansion of the Universe presents cosmology with one of its biggest challenges. While this acceleration can be accommodated by the inclusion of a classical cosmological constant, quantum corrections from vacuum fluctuations are uncontrolled, leading to runaway values which exceed the observed energy-density by many orders of magnitude. A compelling fundamental solution is so far elusive, but cosmologists have proposed a large number of alternative low-energy effective theories, called dark energy models if they inhabit the matter sector and modified gravity if they are in the gravity sector, which aim to cast light on these new forces. While solving the cosmic acceleration problem, such theories alter the growth of structure in the Universe leaving traces of these new forces which may be detectable in galaxy clustering and weak lensing surveys. High accuracy observations are now needed to test the subtle differences in these dark energy and modified gravity theories to constrain the wide range of possibilities and to point the way to a more fundamental theory.

Such high accuracies come from large-scale ground and spacebased surveys, which will provide statistical accuracy but will be limited by their systematic biases. Current weak lensing surveys, such as the VST-Kilo-Degree Survey (KiDS; ${ }^{1}$ Kuijken et al. 2015), the Dark Energy Survey (DES; ${ }^{2}$ Jarvis et al. 2016), Hyper-Suprime Cam (HSC; ${ }^{3}$ Mandelbaum et al. 2018), and future surveys, such as LSST, ${ }^{4}$ Euclid, ${ }^{5}$ and WFIRST, ${ }^{6}$ aim to control their systematics to within the bounds set by the statistical uncertainty. The origin of these biases, and the accuracy to which they can be removed, is being studied at every step of the data analysis, from observation to parameter estimation.

While much effort has gone into trying to correct for biases in weak lensing, the primary approach for correcting biases in current weak lensing is calibration against simulations of the surveys. In CFHTLenS (Heymans et al. 2012; Kilbinger et al. 2013), shear calibration is carried out by estimating the calibration factors as a function of image property from mock weak lensing surveys, and using them to calibrate the 2-point correlation function. In KiDS

\footnotetext{
${ }^{1}$ http://www.astro-wise.org/projects/KIDS

${ }^{2}$ http://www.darkenergysurvey.org

${ }^{3} \mathrm{http}: / /$ www.naoj.org/Projects/HSC/index.html

${ }^{4}$ http://www.lsst.org

${ }^{5} \mathrm{http}: / /$ www.euclid-ec.org, Laureijs, et al. (2011).

${ }^{6} \mathrm{http}: / /$ www.stsci.edu/wfirst/
} 
(Kuijken et al. 2015, Hildebrandt et al. 2017), the shear calibration terms are assumed to be constant and estimated by comparing to simulated mock weak lensing surveys (Fenech Conti et al. 2017). These constant multiplicative bias factors are applied to the shear catalogue, and the calibrated catalogue used to estimate the weak lensing 2-point correlation function (Hildebrandt et al. 2017). Calibration errors are propagated into the parameter estimation assuming a constant error model. The shear 2-point correlation function is in turn used to estimate the shear power spectrum (van Uitert et al. 2018). In the DES survey (Jarvis et al. 2016), calibration factors are also derived from mock surveys and applied directly to the 2-point correlation function.

Theoretical investigations into the effect of systematic biases on weak lensing power spectra has been developing over the last decade (Ishak et al. 2005; Bernstein 2006; Huterer et al. 2006; Knox et al. 2006; Taylor et al. 2007; Amara \& Réfrégier 2008; Kitching, Taylor \& Heavens 2008; Paulin-Henriksson et al. 2008; Kitching et al. 2009; Bernstein \& Huterer 2010; Kitching et al. 2012; Cropper et al. 2013; Massey et al. 2013; Cardone et al. 2014; Kitching et al. 2016). The effect of scale-dependent systematic biases on cosmic shear power was first discussed by Huterer et al. (2006), who studied the impact of a constant multiplicative bias and scaledependent additive image distortions on a range of cosmological parameters, along with the effect of biases in photometric redshifts. Amara \& Réfrégier (2008) investigated the bias due to image distortions arising from a constant multiplicative bias and scale-dependent additive bias, exploring a number of functional forms. They also investigated the effect of redshift-dependence in these biases. These studies found that a constant multiplicative bias on shear should be kept below $\sim 10^{-3}$ and any additive bias shear power should be below $\sim 10^{-10}$ to prevent the bias dominating over noise.

Given that the origin of systematics was still poorly understood Kitching et al. (2009) argued that, instead of choosing a fixed functional form for systematics, one should average over all possible functional forms in a Monte Carlo approach. Building on the work of Paulin-Henriksson et al. (2008), who showed how constant biases in the point spread function (PSF) affect the measurement of shear, Massey et al. (2013) showed how inaccuracies in the PSF, the measurement of galaxy shapes and weighting, and the effect of charge transfer inefficiency (CTI) propagate into the bias and error on the estimated shear. Using the shear power spectrum bias formalism introduced by Kitching et al. (2012) in the GREAT10 Challenge, with both scale-dependent multiplicative and additive shear power bias terms, Massey et al. (2013) averaged over all functional forms for the scale-dependence of the biases and, based on their effects on the dark energy Figure-of-Merit (DEFoM), constrained their amplitude for space-based weak lensing surveys. Cropper et al. (2013) subsequently took these constraints and propagated them back to constraints on individual sources of bias in shear measurement, assuming the bias and uncertainties were independent of scale.

In order to gain a better understand of the source of scaledependence in image distortions, Kitching et al. (2016) simulated systematics in the PSF, CTI and shear estimation, and measured their effect on the shear power spectrum. Using the multiplicative and additive bias power formalism, they propagated this into the bias and the covariance on measured cosmological parameters and found that a survey could minimize the impact of bias by randomizing the observing strategy so that the systematic power became noise-like. They also investigated the removal of sharp spikes in the shear power due to discontinuities on CCD and field-of-view scales.

These simulations demonstrated that many known sources of image distortions will give rise to scale-dependent multiplicative and additive biases. Variations in the shear distortion on the sky correspond to a convolution of the shear signal with the systematic bias in the Fourier domain, rather than a simple multiplicative factor, and so the shear power spectrum will be convolved with the bias power. As a result, if we want to understand how a given potential bias at the image-level propagates into the shear power, or want to know how to forward-model a bias in order to remove it at the level of power spectrum, improved modelling of the shear power spectra is needed. Here, we present a new formalism which accounts for this.

Having identified this convolution-bias effect, we need to know how these biases propagate into the estimation of cosmological parameters and, for survey design, the DEFoM. Past studies have derived constraints on bias effects by comparing the bias in the final cosmological parameter, or Figures-of-Merit, with the expected random error. This assumes that new algorithms can be developed which will mitigate these biases to the level required. However, as we have discussed, the standard approach for data analysis is to calibrate biases with simulated surveys. In this case the relevant factor is the accuracy to which the calibration can be carried out and how this error propagates into dark energy studies. Here, for the first time, we propagate the uncertainty in bias calibration through to parameters and derive requirements based on calibration. As calibration relies on accurate simulations or external data sets we also assess the size of the calibration set required to reach a given accuracy.

The layout of this paper is as follows. We develop a formalism to study how angular variation in systematics arising from image distortions convolve the shear and the inferred convergence field in Section 2, and propagate them into the cosmic shear power spectrum in Section 3. We study how realistic biases in the shear power spectrum can be removed by calibration, marginalizing over the calibration uncertainty. The effect of this on parameter estimation and the impact on the DEFoM is explored in Section 4. Taking realistic examples of image distortions we show how to optimize the constraints on the amplitudes of a set of systematic effects for a given shear survey, to minimize the effect on cosmological parameters in Section 5.

\section{WEAK LENSING BIAS}

The response of a measurement of cosmic shear, $\widehat{\gamma}$, to the true shear field, $\gamma$, in the presence of image distortions can be characterized by a linear model with a local multiplicative factor, $m$, an additive term, $c$ (Heymans et al. 2006; Massey et al. 2007), and a non-local convolution term, $h$, such that

$\widehat{\gamma}=(1+m) \gamma+h * \gamma+c$,

where the shear and bias terms all have angular dependence. The multiplicative factor, $m$, is a spin-2 field producing a change in the amplitude and a local rotation of the shear field, while the additive term is an arbitrary spin-2 shear-like distortion. A multiplicative bias can arise due to miscalibration of the shear measurement caused, for example, by incorrect modelling of the ellipticity or size of the PSF, residual CTI and noise-bias or shear estimation effects. The spin-2 multiplication bias can be written $m(\boldsymbol{\theta})=m_{0}(\boldsymbol{\theta}) \mathrm{e}^{i 2 \phi_{\mathrm{m}}(\boldsymbol{\theta})}$, (Kitching, Taylor \& Heavens 2008) where $\phi_{\mathrm{m}}(\boldsymbol{\theta})$ is the local rotation of the phase and $m_{0}(\boldsymbol{\theta})$ is a local, scalar modulation of the shear amplitude. Massey et al. (2007) and Kitching et al. (2012) investigated a quadratic distortion term, $(1+q) \gamma^{2}$, but found that such second-order terms were negligible. The additive bias, $c$, can arise due to systematics in the ellipticity and shape of the PSF, or from CTI leaving residual streaks in the image. The convolution 
term, $h$, represents a distortion which depends on the shear field at other positions, which may arise due to close packing or blending of galaxy images, and is again a spin-2 field.

Fourier transforming the measured shear field on a flat sky, we find

$\widehat{\gamma}(\boldsymbol{\ell})=\gamma(\boldsymbol{\ell})+\int \frac{\mathrm{d}^{2} \ell^{\prime}}{(2 \pi)^{2}} m\left(\boldsymbol{\ell}-\boldsymbol{\ell}^{\prime}\right) \gamma\left(\boldsymbol{\ell}^{\prime}\right)+c(\boldsymbol{\ell})$,

where the spatially varying multiplicative bias convolves the shear field. For simplicity we have absorbed the convolution distortion, $h$, into the multiplicative bias, $m$. The shear field can also be decomposed on the full curved sky in spherical harmonics (Brown, Castro \& Taylor 2005; Castro, Heavens \& Kitching 2005), but for simplicity we use a flat-sky approximation here. The shear signal can then be decomposed into even-parity convergence modes, $\kappa$, and odd-parity $\beta$ modes $^{7}$ by a rotation of the shear in the Fourier domain, $\kappa(\ell)+i \beta(\ell)=\mathrm{e}^{-2 i \varphi_{\ell}} \gamma(\ell)$, where $\varphi_{\ell}$ is the angle between the wavevector, $\ell$, and an arbitrary axis on the sky. Assuming only a scalar multiplicative bias, $m_{0}$, the measured $\kappa$ and $\beta$ modes are distorted by

$$
\begin{aligned}
\Delta \kappa(\ell)= & \int \frac{\mathrm{d}^{2} \ell^{\prime}}{(2 \pi)^{2}} m_{0}\left(\ell-\ell^{\prime}\right)\left(\kappa\left(\ell^{\prime}\right) \cos 2 \varphi_{\ell \ell^{\prime}}-\beta\left(\ell^{\prime}\right) \sin 2 \varphi_{\ell \ell^{\prime}}\right) \\
& +c_{\kappa}(\ell)
\end{aligned}
$$

$$
\begin{aligned}
\Delta \beta(\ell)= & \int \frac{\mathrm{d}^{2} \ell^{\prime}}{(2 \pi)^{2}} m_{0}\left(\ell-\ell^{\prime}\right)\left(\beta\left(\ell^{\prime}\right) \cos 2 \varphi_{\ell \ell^{\prime}}+\kappa\left(\ell^{\prime}\right) \sin 2 \varphi_{\ell \ell^{\prime}}\right) \\
& +c_{\beta}(\ell),
\end{aligned}
$$

where $\Delta \kappa=\widehat{\kappa}-\kappa$ and $\Delta \beta=\widehat{\beta}-\beta$ are the changes in the convergence and $\beta$ fields, and $\varphi_{\ell \ell^{\prime}}=\varphi_{\ell}-\varphi_{\ell^{\prime}}$ is the angle between the Fourier modes. We have also decomposed the additive bias into even- and odd-parity modes, where $c_{\kappa}(\ell)+i c_{\beta}(\ell)=\mathrm{e}^{-2 i \varphi \ell} c(\ell)$. The mode-mixing effect of the full spin-2 multiplicative bias is slightly more complex and we present full expressions in Appendix A. As discussed by Kitching et al. (2012), the effect of a spatially varying multiplicative bias is similar to that of a survey mask in the Pseudo-Cl (PCL) power spectrum formalism (Hivon et al. 2002) for CMB polarization (Brown, Castro \& Taylor 2005), and so we can easily generalize our results to a masked survey.

\section{COSMIC SHEAR POWER}

The correlations of the different Fourier modes of the shear fields, $(X, Y)=(\kappa, \beta)$, for different $\ell$ modes is given by

$$
\left\langle X(\ell) Y^{*}\left(\ell^{\prime}\right)\right\rangle=(2 \pi)^{2} C^{X Y}(\ell) \delta_{\mathrm{D}}\left(\ell-\ell^{\prime}\right),
$$

where $C^{X Y}(\ell)$ is the convergence, $\beta$-mode and cross-power spectrum. We assume all fields are statistically homogeneous and isotropic on a flat sky, and $\delta_{\mathrm{D}}(\ell)$ is the Dirac delta function. The measured convergence power spectrum on a flat, finite patch of sky of area, $A$, is given by

$\widehat{C}^{\kappa \kappa}(\ell)=\frac{1}{A}\left\langle|\widehat{\kappa}(\ell)|^{2}\right\rangle$,

where we have approximated the zero-lag delta function by $\delta_{\mathrm{D}}(\mathbf{0})=$ $A /(2 \pi)^{2}$.

\footnotetext{
${ }^{7}$ In this paper, we shall refer to $B$ modes in lensing as $\beta$ modes, where $E$ modes correspond to the convergence field, $\kappa$.
}

We denote the systematic bias fields on the sky by $Z(\boldsymbol{\theta})=$ $\left(m, c_{\kappa}, c_{\beta}\right)$ for each of the multiplicative/convolution fields and the even- and odd-parity modes of the additive biases. These biases can be split into a constant term across the survey, $Z_{0}=b_{Z}$, a spatially varying deterministic bias, $\Delta Z(\theta)$, around the mean which can arise from variations which can be modelled by a template, and a stochastic term, $\delta Z(\theta)$, that arise from either noise in the measurement of the bias or other indeterminate aspects of the bias that can only be modelled statistically (e.g. Massey et al. 2013); hence $Z=b_{Z}+\Delta Z+\delta Z$. The correlations of the Fourier modes of the fluctuating part of the bias are given by

$\left\langle\delta Z(\ell) \delta Z^{*}\left(\ell^{\prime}\right)\right\rangle=(2 \pi)^{2} \mathcal{C}_{Z}(\ell) \delta_{\mathrm{D}}\left(\boldsymbol{\ell}-\boldsymbol{\ell}^{\prime}\right)$,

where $\mathcal{C}_{Z}(\ell)$ is the power spectrum of the bias fluctuations. The assumption of statistical isotropy can be relaxed to allow for anisotropic effects such as, for example, from CTI or other effects aligning with the CCD pixels, and with other directional dependences.

Taking equations (3) and (4), and using equations (5) and (7), we can calculate the correlators of the measured convergence and $\beta$ modes. We present the full correlations of the observed Fourier modes of the convergence and $\beta$ fields for an arbitrary spin- 2 multiplicative bias in Appendix B, equations (B1) to (B3), from which we see that the observed convergence power is

$$
\begin{aligned}
\widehat{C}^{\kappa \kappa}(\ell)= & \left(1+b_{\mathrm{m}}\right)^{2} C^{\kappa \kappa}(\ell) \\
& +\int \frac{\mathrm{d}^{2} \ell^{\prime}}{(2 \pi)^{2}} \mathcal{G}\left(\ell-\ell^{\prime}\right) C^{\kappa \kappa}\left(\ell^{\prime}\right)+\mathcal{A}^{\kappa \kappa}(\ell),
\end{aligned}
$$

where $b_{\mathrm{m}}$ is a constant multiplicative bias, the convolution kernel is

$$
\mathcal{G}\left(\boldsymbol{\ell}-\boldsymbol{\ell}^{\prime}\right)=\left(\frac{1}{A}\left|\Delta b_{\mathrm{m}}\left(\boldsymbol{\ell}-\boldsymbol{\ell}^{\prime}\right)\right|^{2}+\mathcal{C}_{\mathrm{m}}\left(\boldsymbol{\ell}-\boldsymbol{\ell}^{\prime}\right)\right) \cos ^{2} 2 \varphi_{\ell \ell^{\prime}},
$$

and the additive bias is $\mathcal{A}^{\kappa \kappa}(\ell)=\left|\Delta b_{c_{\kappa}}(\ell)\right|^{2} / A+\mathcal{C}_{c_{\kappa}}(\ell)$. This relationship between the observed and true convergence is a central result of our paper. Any constant additive bias appears only in the $\ell=0$ mode and can be ignored. Similar expressions for the $\beta$-mode power spectrum are derived in Appendix C. From equation (8) we see that the constant bias over the survey, $b_{\mathrm{m}}$, factors out into a multiplicative bias of the convergence power spectrum. The scale-dependence of the multiplicative bias, $\Delta b_{\mathrm{m}}(\ell)$, comes in only through the convolution term at second-order, and is weighted by the inverse survey area. Finally, the power spectrum of the indeterminate stochastic bias, $\mathcal{C}_{\mathrm{m}}(\ell)$, is also convolved with the convergence power spectrum, while the additive power is composed of the square of the additive shear bias variation and its power spectrum.

In a number of applications to data (e.g. Hildebrandt et al. 2017), and in theoretical models (e.g. Huterer et al. 2006; Amara \& Réfrégier 2008) the multiplicative bias is assume to be a constant while the additive bias may have scale-dependence. If we model the multiplicative bias by a constant mean value, $b_{\mathrm{m}}$, and random fluctuations about this with variance $\sigma_{\mathrm{m}}^{2}$, but keep the additive bias as scale dependent we find

$\widehat{C}^{\kappa \kappa}(\ell)=\left(\left(1+b_{\mathrm{m}}\right)^{2}+\frac{1}{2} \sigma_{\mathrm{m}}^{2}\right) C^{\kappa \kappa}(\ell)+\mathcal{A}^{\kappa \kappa}(\ell)$.

In this case the convolution by the spatially varying multiplicative bias has become a constant multiplicative bias factor, $\left(1+b_{\mathrm{m}}\right)^{2}+$ $\sigma_{\mathrm{m}}^{2} / 2$, where the variance in the bias now adds to the shear power bias with a factor $\sigma_{\mathrm{m}}^{2} / 2$. The other half of the variance contributes to the odd-parity $\beta$-mode power (see Appendix $\mathrm{C}$, equation $\mathrm{C} 4$ ). In Fig. 1, we show this model (the lower solid dark grey, purple in 

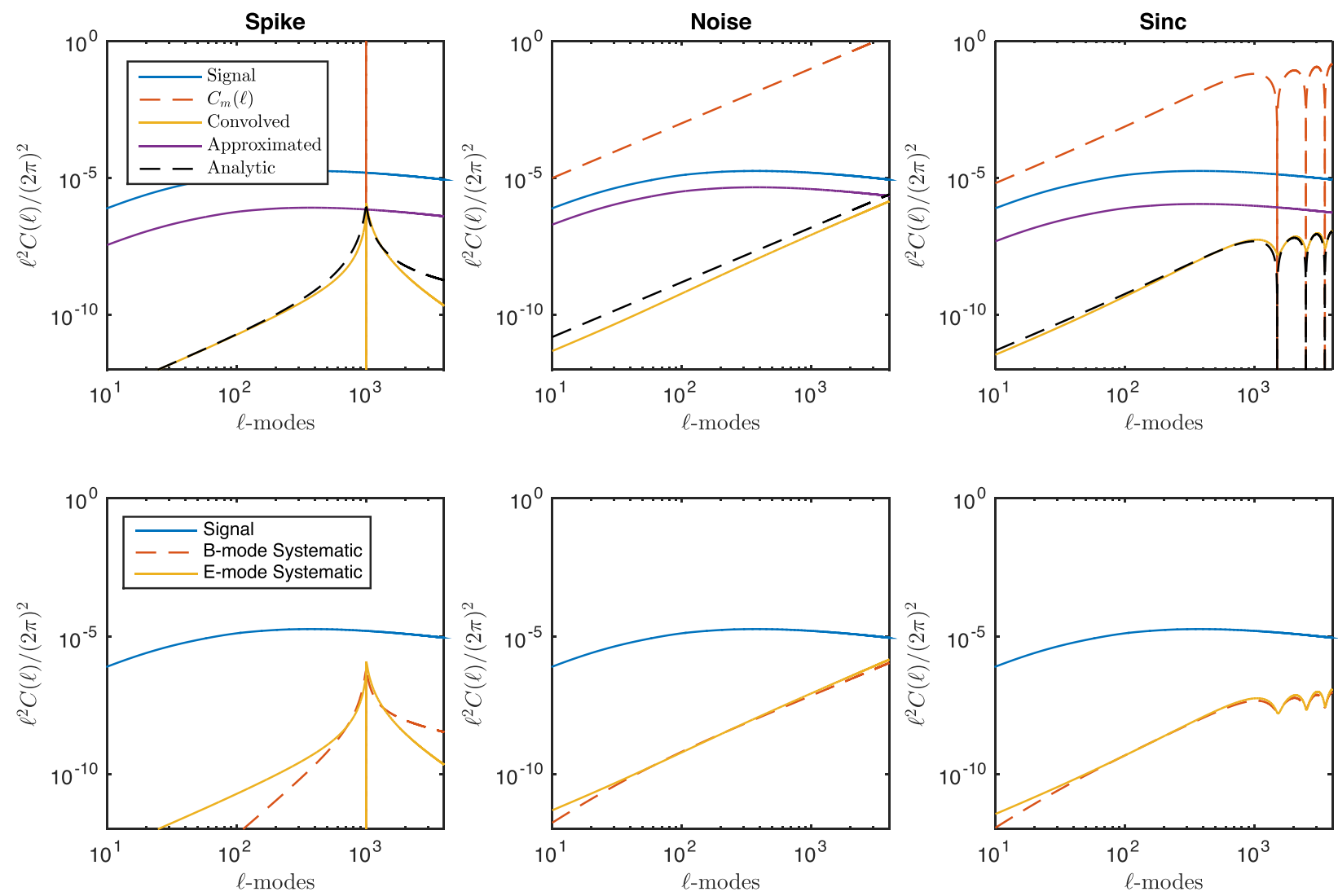

Figure 1. Convolution shear-convergence and $\beta$-mode power bias. In all panels the upper solid curve (blue online) are the true convergence power spectrum signal, $C_{\ell}^{\kappa \kappa}$; in the top panels the upper dashed line (red online) are the multiplicative noise power, $\mathcal{C}_{\mathrm{m}}(\ell)$; lower solid lines (yellow online) are the bias power numerically convolved with the convergence power, while the black dashed lines are the exact analytic convolution from Section 3 , which are in good agreement with the numerical convolutions. Upper curves below the true shear power (purple online) are the constant bias given by equation (10), which is scale independent. In the lower plots the lower solid lines (yellow online) are again the E-mode bias power spectra and orange lower dashed lines (red online) are the $\beta$-mode power generated by mode mixing. Left-hand panels: The convergence power is convolved with a sharp bias feature at $\ell=10^{3}$, leading to an extended bias power, with good agreement between numerical and analytic convolutions. $\beta$-mode power is generated from power at $\ell_{0}$ (lower plot). Middle panels: A constant noise-like bias leads to a constant convolution bias, suppressed by the shear variance, as predicted by the analytic results. A constant $\beta$-mode power spectrum is generated with the same amplitude as the bias convergence power (lower panel). Right-hand panels: A sinc-like bias power is convolved with the convergence power, where the shape and amplitude is well approximated by the analytic model of Section 3 . We find a $\beta$-mode power of equal shape and amplitude (lower panel).

online version, curves in the upper panels) along with other model bias power spectra.

Following Kitching et al. (2016), we can model the detailed effect of more realistic bias power spectra. If the bias power spectrum is a sharp peak at $\ell=\ell_{0}$, we can approximate it by a delta-function, $\mathcal{C}_{\mathrm{m}}(\ell)=(2 \pi)^{2} \mathcal{C}_{0} \delta_{\mathrm{D}}\left(\left|\ell-\boldsymbol{\ell}_{0}\right|\right) / \ell$. The change in the measured shear power is then $\Delta \widehat{C}^{\kappa \kappa}(\ell)=\mathcal{C}_{0} C^{\kappa \kappa}\left(\left|\ell-\ell_{0}\right|\right)$, which shifts the shear power origin to $\ell_{0}$, reflects about it and rescales the amplitude. A spike of power centred at wavevector $\ell=\ell_{0}$ would not induce $\beta$ modes, but here we have assumed an isotropic distribution localized about a single wavelength which will generate $\beta$ modes of similar amplitude. For a constant noise-like bias power, $\mathcal{C}_{\mathrm{m}}(\ell)=C_{\mathrm{N}}$, the change in the shear power is $\Delta \widehat{C}_{\ell}^{\kappa \kappa}=C_{\mathrm{N}} \sigma_{\kappa}^{2} / 2$, where the other half of the convolved noise power has been converted into a $\beta$-mode power spectrum (see Appendix C). Kitching et al. (2016) also found that sharp features in a weak lensing survey associated with biases on particular scale, $\theta$, such as the field of view, resulted in a sinc-like scale-dependent bias power, $C_{\mathrm{S}}(\ell \theta) \propto \operatorname{sinc}(\ell \theta)$. Since this is noise-like on large scales, to a good approximation the change in the shear power is $\Delta \widehat{C}^{\kappa \kappa}(\ell)=C_{\mathrm{S}}(\ell \theta) \sigma_{\kappa}^{2} / 2$, where again a $\beta$-mode power of equal amplitude is generated.
Fig. 1 shows the shear-convergence power for a $\Lambda$ CDM model with parameters taken from Planck (Planck Collaboration XIII 2016) along with the three generic, multiplicative scale-dependent biases: a localized spike in bias power with $\mathcal{C}_{0}=1$, which may arise from residual corrections on a particular scale (Fig. 1, left-hand panels); a constant noise-like power bias with $C_{\mathrm{N}}=10^{-10}$, which may arise from random variations in a residual bias across the survey on all scales (Fig. 1, middle panels); and a sinc-like bias function (Fig. 1, right-hand panels) of the form $C_{\mathrm{S}}\left(\ell \theta_{\mathrm{s}}\right)=10^{-10} \operatorname{sinc}\left(\ell \theta_{\mathrm{S}}\right)$, where $\theta_{\mathrm{s}}=10^{-3} \mathrm{rad}$, which may arise due to a residual bias over a finite patch such as the CCD of field-of-view scales (Kitching et al. 2016). The convolution of each of these multiplicative biases with the shear power is also shown in each panel (lower solid line, yellow online), along with the analytic expressions found above (black, dashed lines). In all cases we find that the analytic formula predicts the numerical data well.

As expected the local bias spike leads to a bias spread out in $\ell$-space, although still highly peaked and centred around the spike, while the noise-like bias power convolution gives rise to a constant bias whose amplitude is suppressed by half of the shear-convergence variance, $\sigma_{\kappa}^{2} / 2$. The convolved sinc-like power has the same form 
as the bias power, also suppressed by a factor of half the shearconvergence variance. There is an induced $\beta$-mode power for the sharp spike (Fig. 1, lower left panel), while the $\beta$-mode noise power is equal to the convergence noise power (Fig. 1, lower middle panel). We also find the $\beta$-mode power from the sinc bias is the same as the convergence power (Fig. 1, lower right panel), as predicted.

We note that in the past the effects of multiplicative bias on the shear power spectra has been assumed to be a scale-dependent multiplicative term in Fourier space (Amara \& Réfrégier 2008; Kitching et al. 2012; Cropper et al. 2013; Massey et al. 2013; Kitching et al. 2016),

$\widehat{C}^{\kappa \kappa}(\ell)=[1+\mathcal{M}(\ell)] C^{\kappa \kappa}(\ell)+\mathcal{A}^{\kappa \kappa}(\ell)$.

This would only be appropriate if the biases were dominated by convolution terms in real-space, $h$ in equation (1). As we expect image effects to have a multiplicative angular dependence on the sky, our convolution model for the biased power is more realistic and will correctly model the response of the shear power to realistic biases. We recommend our new results, equations (8) and (9), should be used in future modelling.

\section{CALIBRATION AND REMOVAL OF BIAS}

In previous studies, the effect of systematics in cosmic shear measurements has focused on propagating the biases into the dark energy parameters and setting constraints such that either the biases are less than the measurement error (e.g. Amara \& Réfrégier 2008), or that the DEFoM is kept above some fixed value (e.g. Massey et al. 2013). This is useful if there is an algorithmic way to remove these biases. However, in practice many systematics may be too complex to model to sufficient accuracy, and so they need to be removed by calibration to external data or simulations and the uncertainty on the calibration then marginalized over (e.g. Hildebrandt et al. 2017). This suggests that it is not sufficient to know how the bias affects the dark energy measurement - we also need to know how marginalization over the uncertainty in the calibration propagates into the measurement.

We can explore the effect of calibration and marginalization using the Fisher matrix formalism (e.g. Tegmark, Taylor \& Heavens 1997). Let us assume the measured shear-convergence power, $\widehat{C}_{\ell}^{\kappa \kappa}(\boldsymbol{\theta})$, given by equations (8) and (9), is Gaussian distributed, $\mathcal{P}\left(\widehat{C}_{\ell}^{\kappa \kappa} \mid \boldsymbol{\theta}\right)$, and depends on a set of cosmological parameters, $\boldsymbol{\theta}$, whose likelihood function, $\mathcal{L}(\boldsymbol{\theta})=\mathcal{P}\left(\widehat{C}_{\ell}^{\kappa \kappa} \mid \boldsymbol{\theta}\right)$, is also Gaussian distributed in parameter space. The expected cosmological parameter covariance matrix for this likelihood is $C=\left\langle\Delta \boldsymbol{\theta} \Delta \boldsymbol{\theta}^{t}\right\rangle=$ $\mathcal{F}^{-1}$, where

$\mathcal{F}_{\alpha \beta}=\frac{4 \pi f_{\text {sky }}}{2} \int \frac{\ell \mathrm{d} \ell}{2 \pi}\left[\widehat{C}^{\kappa \kappa}(\ell)+N\right]^{-2} \frac{\partial \widehat{C}^{\kappa \kappa}(\ell)}{\partial \theta_{\alpha}} \frac{\partial \widehat{C}^{\kappa \kappa}(\ell)}{\partial \theta_{\beta}}$,

is the Fisher matrix, $A=4 \pi f_{\text {sky }}$ is the area of the survey, and $N=2 \pi f_{\text {sky }} \sigma_{\mathrm{e}}^{2} / N_{\mathrm{g}}$ is the shear noise power for $N_{\mathrm{g}}$ galaxies with intrinsic ellipticity dispersion $\sigma_{\mathrm{e}}$.

We assume the convolved and additive bias power are functions of a set of bias parameters, $\boldsymbol{\psi}$, so that $\widehat{C}_{\ell}^{\kappa \kappa}(\boldsymbol{\theta}, \boldsymbol{\psi})$ now depends on $\boldsymbol{\psi}$ and has the distribution $\mathcal{P}\left(\widehat{C}_{\ell}^{\kappa \kappa} \mid \boldsymbol{\theta}, \boldsymbol{\psi}\right)$. These bias parameters can be estimated from external data or simulations, with the distribution $\mathcal{P}\left(\boldsymbol{\psi} \mid \boldsymbol{\psi}_{0}, \widehat{C}_{\psi \psi^{\prime}}\right)$, with mean given by the true bias values, $\langle\boldsymbol{\psi}\rangle=\boldsymbol{\psi}_{0}$, and covariance matrix, $\widehat{C}_{\psi \psi^{\prime}}$. We assume this distribution is also Gaussian. The biased shear power distribution can now be corrected by marginalizing over the calibration measurement distribution,

$\mathcal{P}\left(\widehat{C}_{\ell}^{\kappa \kappa} \mid \boldsymbol{\theta}, \boldsymbol{\psi}_{0}, \widehat{C}_{\psi \psi^{\prime}}\right)=\int \mathrm{d} \psi \mathcal{P}\left(\widehat{C}_{\ell}^{\kappa \kappa} \mid \boldsymbol{\theta}, \boldsymbol{\psi}\right) \mathcal{P}\left(\boldsymbol{\psi} \mid \boldsymbol{\psi}_{0}, \widehat{C}_{\psi \psi^{\prime}}\right)$,

which will correct the bias and widen the shear likelihood.

Expanding the observed shear-convergence power to first order in the bias parameters, we can carry this marginalization out analytically (Taylor \& Kitching 2010). The cosmological parameter covariance matrix from this marginalized likelihood can be found from the inverse of the marginalized Fisher matrix, $C^{\mathrm{M}}=\left[\mathcal{F}^{\mathrm{M}}\right]^{-1}$, where the marginalized Fisher matrix is given by the Schur compliment of the cosmological and bias parameter Fisher matrix,

$\mathcal{F}_{\theta \theta^{\prime}}^{\mathrm{M}}=\mathcal{F}_{\theta \theta^{\prime}}-\mathcal{F}_{\theta \psi}\left[\mathcal{F}_{\psi \psi^{\prime}}+\widehat{C}_{\psi \psi^{\prime}}^{-1}\right]^{-1} \mathcal{F}_{\psi^{\prime} \theta^{\prime}}$

As the accuracy of the external calibration increases, the second term vanishes and the parameter variance is unchanged. However, even if the external calibration is removed the loss of accuracy in the parameters is finite, because we can self-calibrate the biases using the cosmic shear survey itself.

As well as the effect on the cosmological parameter covariance matrix, we can also estimate the effect on the DEFoM. The DEFoM is defined as the inverse area of the 68.3 per cent confidence region of the dark energy 2-parameter space, $w=\left(w_{0}, w_{a}\right)$, after marginalizing over all other cosmological parameters (Albrecht et al. 2006). For Gaussian distributed parameters this is given by the determinant of the dark energy Fisher matrix,

$\mathrm{F}_{\mathrm{oM}}^{\mathrm{DE}}=\operatorname{det} \mathcal{F}_{w w^{\prime}}^{\mathrm{DE}}$,

where $\mathcal{F}_{w w^{\prime}}^{D E}$ is the dark energy parameter Fisher matrix found by marginalizing the cosmological parameter space over all other cosmological parameters. This is also given by the Schur complement of the full parameter Fisher matrix,

$\mathcal{F}_{w w^{\prime}}^{\mathrm{DE}}=\mathcal{F}_{w w^{\prime}}-\mathcal{F}_{w \theta} \mathcal{F}_{\theta \theta^{\prime}}^{-1} \mathcal{F}_{\theta^{\prime} w^{\prime}}$,

where, in this expression, $\theta$ are all the cosmological parameters excluding the dark energy $w$-vector. The effect of calibration and marginalization on the DEFoM can be calculated by replacing the Fisher matrices in equation (16) with marginalized ones from equation (14).

We can estimate the effect of calibration on the DEFoM. The fractional change in the DEFoM from a change in the Fisher matrix, to first order, is

$\Delta \ln \mathrm{F}_{\mathrm{oM}}^{\mathrm{DE}}=\operatorname{Tr}\left(\Delta \mathcal{F}^{\mathrm{DE}}\left[\mathcal{F}^{\mathrm{DE}}\right]^{-1}\right)$,

where $\Delta \mathcal{F}_{w w^{\prime}}^{\mathrm{DE}}=\Delta \mathcal{F}_{w w^{\prime}}-\Delta\left[\mathcal{F}_{w \theta} \mathcal{F}_{\theta \theta^{\prime}}^{-1} \mathcal{F}_{\theta^{\prime} w^{\prime}}\right]$. Following marginalization over the calibration parameters, $\psi$, using equation (14), the fractional change in the DEFoM is

$\Delta \ln \mathrm{F}_{\mathrm{oM}}^{\mathrm{DE}}=-\left(\mathcal{F}_{\psi w}^{\mathrm{M}} \mathcal{F}_{w w^{\prime}}^{-1} \mathcal{F}_{w^{\prime} \psi^{\prime}}^{\mathrm{M}}\right)\left[\mathcal{F}_{\psi^{\prime} \psi}+\widehat{C}_{\psi^{\prime} \psi}^{-1}\right]^{-1}$,

where $\mathcal{F}_{\psi w}^{\mathrm{M}}=\mathcal{F}_{\psi w}-\mathcal{F}_{\psi \theta} \mathcal{F}_{\theta \theta^{\prime}}^{-1} \mathcal{F}_{\theta^{\prime} w}$ is the joint Fisher matrix of dark energy and calibration parameters, marginalizing over all other cosmological parameters. Equation (18) shows explicitly the relationship between the calibration accuracy and degradation of the DEFoM. Again, if the accuracy of external calibration is high the DEFoM is unchanged, while if it is removed, self-calibration limits the reduction in the DEFoM. 
In dark energy studies, we usually want the absolute contribution from the fractional DEFoM bias to be less than some threshold, $v$, so that

$\left|\Delta \ln \mathrm{F}_{\mathrm{OM}}\right| \leq v$.

As an example, if we consider a constant dark energy equation-ofstate parameter, $w=w_{0}$, and one other cosmological parameter, $\theta$, and a single, constant multiplicative calibration parameter (equation $10), \psi=\mathcal{M}=2 b_{\mathrm{m}}+b_{\mathrm{m}}^{2}$, with covariance $\widehat{C}_{\mathcal{M} \mathcal{M}^{\prime}}=\widehat{\sigma}_{\mathcal{M}}^{2} I_{\mathcal{M} \mathcal{M}^{\prime}}$, the fractional decrease in the DEFoM is

$\Delta \ln \mathrm{F}_{\mathrm{oM}}^{\mathrm{DE}}=-\left|\Delta \mu_{\mathcal{M} w_{0}}\right|^{2}\left(\frac{\widehat{\sigma}_{\mathcal{M}}^{2}}{\widehat{\sigma}_{\mathcal{M}}^{2}+\sigma_{\mathcal{M}}^{2}}\right)$,

where $\Delta \mu_{\alpha \beta}=\mu_{\alpha \beta}-\mu_{\alpha \theta} \mu_{\theta \beta}$, with the implied summation over all other parameters, $\theta ; \mu_{\alpha \beta}=\mathcal{F}_{\alpha \beta} / \sqrt{\mathcal{F}_{\alpha \alpha} \mathcal{F}_{\beta \beta}}$ is the Fisher matrix correlation coefficient; $\sigma_{\mathcal{M}}^{2}=1 / \mathcal{F}_{\mathcal{M M}}$ is the self-calibration variance of $\mathcal{M}$ measured from the survey itself; and $\widehat{\sigma}_{\mathcal{M}}^{2}$ is the external calibration variance. The decrease in the DEFoM vanishes as the external error on $\mathcal{M}$ vanishes, while for no external calibration the fractional change is equal to $-\left|\Delta \mu_{\alpha \beta}\right|^{2}$ and determined by the correlation of the bias parameters with the dark energy and cosmological parameters.

The variance of $\mathcal{M}$ estimated from the survey is $\sigma_{\mathcal{M}}^{2}=1 / N_{\text {eff }}$, where $N_{\text {eff }}$ is the effective number of independent modes measured in the shear power spectrum. If we require the contribution to the DEFoM from bias calibration to be less than 10 per cent, so that $v$ $\leq 0.1$, and assume that the number of effective modes measured in the shear power spectrum is $N_{\text {eff }} \approx 10^{5}$, and $\left|\Delta \mu_{\mathcal{M} w_{0}}\right| \approx 1$, then the error on the multiplicative calibration needs to be less than 0.1 per cent, or $\widehat{\sigma}_{\mathcal{M}}<10^{-3}$. However, if the Fisher correlation coefficient is less than unity this constraint will weaken. Similarly the fractional bias in the DEFoM from a constant additive bias has the same form as equation (20) with $\mathcal{M} \rightarrow \mathcal{A}$, where $\sigma_{\mathcal{A}}^{2}=$ $\mathcal{F}_{\mathcal{A} \mathcal{A}}^{-1}=\Delta \bar{C}$ is the inverse-weighted mean power, which implies the calibration error on the additive bias power calibration should be $\widehat{\sigma}_{\mathcal{A}}<10^{-12}$.

As well as constant biases, we can investigate the more realistic cases of the spike, noise, and sinc-like bias functions. Using the bias power functions introduced in Section 3 and illustrated in Fig. 1 with the same parameters, we can add them together to form a multiplicative/convolved bias and use the same function as an additive bias power. We can then vary the accuracy with which we can measure the amplitude of the multiplicative/convolved and additive bias powers, to see its effect on the change in the DEFoM. Fig. 2 shows the fractional change in the DEFoM as a function of the variance of the external calibration for constant multiplicative and additive biases from a numerical calculation of the DEFoM. As expected, as the external calibration accuracy decreases, the DEFoM is reduced and tends to a constant beyond $\Delta \ln F_{\mathrm{FoM}}^{\mathrm{DE}}=-0.3$, when the bias is self-calibrated by the survey. For an accurate calibration of the additive bias amplitude, we find the calibration accuracy on the multiplicative/convolution bias roughly agrees with our naive estimate, where a 10 per cent change, $\Delta \ln F_{\mathrm{FoM}}^{\mathrm{DE}}=-0.1$, requires a calibration error of around $\widehat{\sigma}_{\text {multi }} \approx 0.07$, estimated from Fig. 2 . However, the additive calibration error is much higher at around $\widehat{\sigma}_{\text {add }} \approx 10^{-8}$. Both of these constraints from calibration appear to be weaker than the constraints derived from requiring an algorithmic bias correction (e.g. Massey et al. 2013).

Fig. 3 shows the change in the DEFoM due to the calibration of each individual additive and multiplicative (convolution) bias spectra for spike, noise, and sinc-like functions, as a function of the

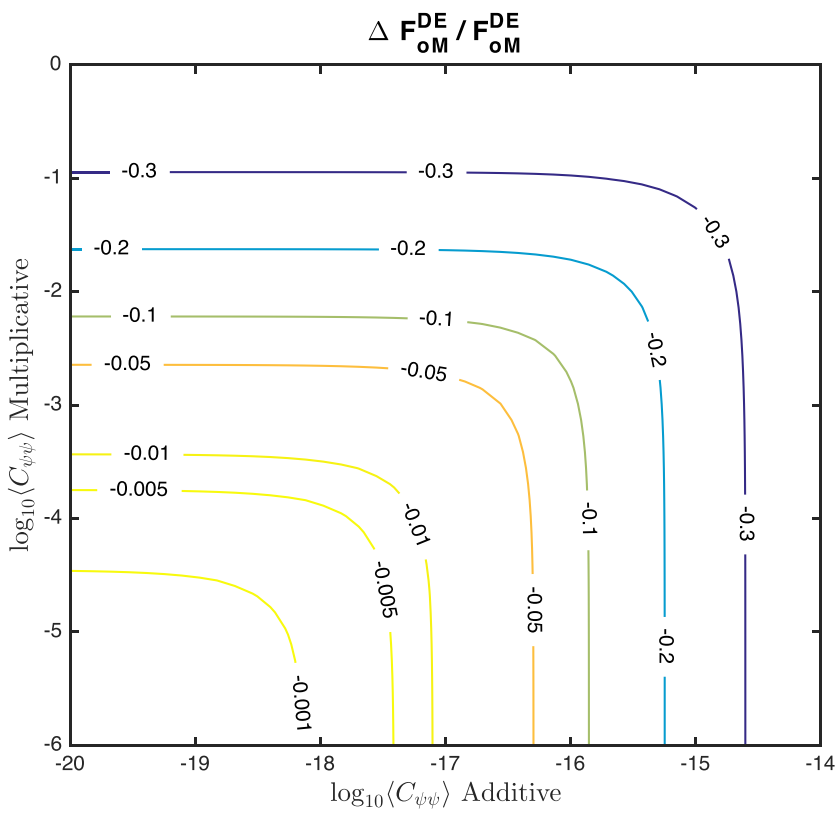

Figure 2. The fractional decrease in the DEFoM, $\Delta \ln F_{\mathrm{oM}}^{\mathrm{DE}}$, as a function of the error on the external calibration of an additive bias and multiplicative (convolution) bias, owing to calibration and marginalization. The functional form of the bias is the sum of the spike, noise, and sinc functions shown in Fig. 1, and we assume only the amplitude requires calibration. The axes are the external calibration variance, $\widehat{C}_{\psi \psi}$, where the indices $\psi$ are the amplitudes of the multiplicative (convolutions) and additive biases. Beyond $\Delta \ln F_{\mathrm{oM}}^{\mathrm{DE}}=-0.3$ the surface flattens as we reach the self-calibration regime.

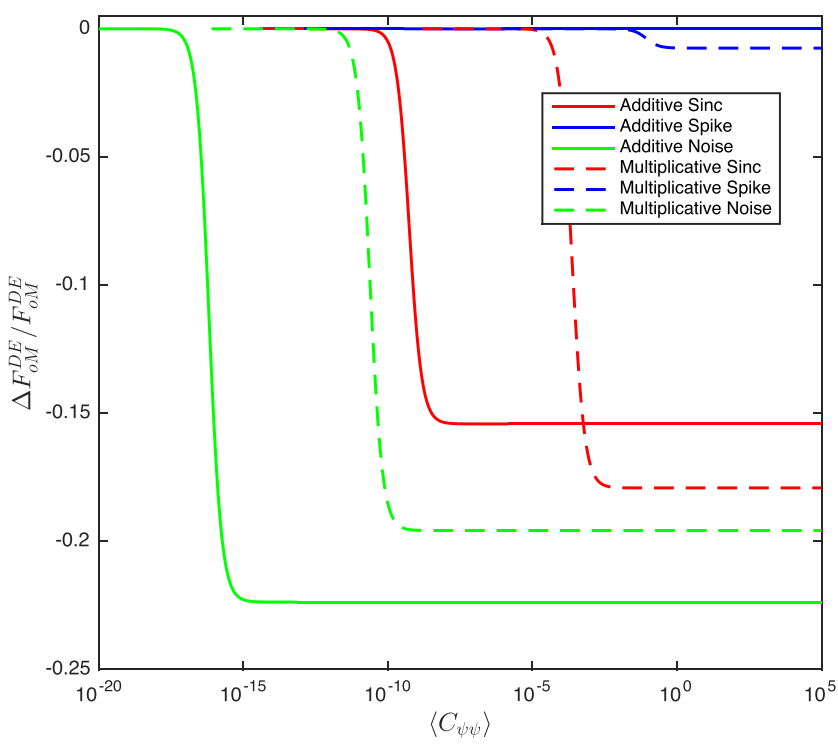

Figure 3. The fractional decrease in the DEFoM, $\Delta \ln F_{\mathrm{FoM}}^{\mathrm{DE}}$, due to noiselike (from left the first solid and dashed lines, green online), sinc-like (the middle solid and dashed line, red online), and spike (the right and upper two solid and dashed lines, blue online) bias power calibration, removal, and marginalization, as a function of the prior calibration error in the bias power amplitude. Solid lines are for additive biases, while dashed lines are for convolutions biases. For a small external calibration error the change in $\Delta \ln F_{\mathrm{FoM}}^{\mathrm{DE}}$ vanishes. For larger bias error the DEFoM decreases until the data itself calibrates the bias at the cost of constraints on other cosmological parameters. 
external calibration error on each bias calibration. Again, we assume only the amplitude is to be calibrated with a fixed functional form.

The additive spike bias (right upper solid and dashed lines, blue online) has the smallest effect on the DEFoM, because the addition of a spike in the shear power has very little correlation with cosmological parameters. This agrees with the analysis of Kitching et al. (2016), who found a significant bias from an additive spike, but little increase in the $w_{0}, w_{a}$ error ellipse (see Fig. 1 of Kitching et al., 2016). However, convolution with the shear power extends this over a range of wavenumbers, and it acquires a cosmological dependence (see Fig. 1, left-hand panel). Removal of this bias requires only a modest accuracy, $\widehat{\sigma}_{\text {spike, add }} \approx 1$. As this cosmological dependence still does not mimic the effects on the true shear power, self-calibration with a shear survey works well, resulting in only a small reduction in the DEFoM.

The noise bias (left lower solid and dashed lines, green online) has the highest calibration requirements, with the additive bias (most left solid line) requiring a high accuracy of $\widehat{\sigma}_{\text {noise, add }}<10^{-8}$ for removal, while self-calibration leaves the largest reduction in the DEFoM. This can be understood from the noise power having greatest effect at high wavenumber, where the dark energy equation of state will have greatest effect. The multiplicative/convolution bias also requires a high accuracy to calibrate, as the resulting convolution is still noise-like and has a cosmological dependence.

The sinc-function bias power is similar to the noise-like bias power, with an effect on the DEFoM between the noise and spike bias power, with the oscillations dampening the bias power at high wavenumber, which de-correlates the bias and cosmological parameters. Again, the additive sinc bias requires more accurate calibration than the multiplicative/convolution bias. Finally, we find the accuracy of the calibration scales with the amplitude of the bias, such that numerically we find $\widehat{C}_{\psi \psi}(\psi) \propto \psi^{-1}$, for all biases.

In summary, we find the calibration and removal of noise-like biases has the greatest impact on dark energy studies, followed by sinc-like biases. The effect of calibration and removal of spikes in the shear power spectrum has the least effect. Caveats to this study are that we consider only calibration of the amplitude of these bias effects. In detail, for the spike and sinc bias functions, we would also want to calibrate the scale at which the bias occurs, while the functional forms of the systematic power may require many more parameters to describe.

\section{OPTIMIZING BIAS CALIBRATION}

Since we can expect the calibration of any shear bias has a cost, either in the collection of external calibration data or the generation of realistic simulations, it is useful to have a guide for where to optimally allocate resources in investigating sources of bias, their calibration and removal. Here, we shall assume that any bias can be modelled through a simulation of the experiment, and that the accuracy on the measurement of any bias is limited only by the number of simulations that can be generated. A similar calculation can be done if the cost of the calibration arises from collecting external data. We assume these biases are independent, and work to first order in the external calibration error, $\widehat{\sigma}_{\psi}^{2}$, so that the effect on the DEFoM can be written $\Delta \ln \mathrm{F}_{\mathrm{oM}}^{\mathrm{DE}}=-\Phi_{\psi} \widehat{\sigma}_{\psi}^{2}$, where $\Phi_{\psi}=\mathcal{F}_{\psi w}^{M} \mathcal{F}_{w w^{\prime}}^{-1} \mathcal{F}_{w^{\prime} \psi}^{\mathrm{M}}$ and there is no summation over repeated $\psi$ in this last expression. These approximations mean that we are working to order-of-magnitude accuracy. In addition, more simulations may be needed to explore the effects of possible biases or correlations between biases, and so our estimate here is a lower bound on the number of simulations, or size of data set, required for calibration.

If we further assume that each bias requires its own set of simulations for calibration, the total number of simulations needed to calibrate all biases with an accuracy of $\widehat{\sigma}_{\psi}^{2}$ scales as

$N_{\mathrm{S}}=\sum_{\psi} \frac{\alpha_{\psi}}{\widehat{\sigma}_{\psi}^{2}}$

where $\alpha_{\psi}$ is a parameter which normalizes the number of simulations needed to calibrate each bias and depends on the properties of the bias. Our aim is to minimize the number of simulations needed for calibration, with the constraint that we do not exceed the desired fractional change in the DEFoM, $v=\left|\Delta \ln \mathrm{F}_{\mathrm{oM}}^{\mathrm{DE}}\right|$. We can calculate this by minimizing the merit function,

$S=N_{\mathrm{S}}+\lambda \nu$,

with respect to $\widehat{\sigma}_{\psi}^{2}$, where $\lambda$ is a Lagrangian multiplier to constrain the DEFoM. Minimizing this with respect to the measured external calibration error, $\widehat{\sigma}_{\psi}^{2}$, taking note that $v$ is the absolute value of the fractional change in the DEFoM, and using the identity $|x|=\sqrt{x^{2}}$, we find

$\widehat{\sigma}_{\psi}^{2}=\left|\frac{\alpha_{\psi}}{\lambda \Phi_{\psi}}\right|^{1 / 2}$.

Using the constraint on the DEFoM we can replace the Lagrangian multiplier, $\lambda$, with $v$, to find that the minimum number of simulations is

$N_{\mathrm{S}}=\left.\frac{1}{v}\left(\sum_{\psi^{\prime}} \alpha_{\psi^{\prime}}\left|\frac{\Phi_{\psi^{\prime}}}{\alpha_{\psi^{\prime}}}\right|^{1 / 2}\right)\left|\sum_{\psi^{\prime \prime}} \Phi_{\psi^{\prime \prime}}\right| \frac{\alpha_{\psi^{\prime \prime}}}{\Phi_{\psi^{\prime \prime}}}\right|^{1 / 2} \mid$,

which yields the error on the bias calibration,

$\widehat{\sigma}_{\psi}^{2}=v\left|\frac{\alpha_{\psi}}{\Phi_{\psi}}\right|^{1 / 2}\left|\left(\sum_{\psi^{\prime}} \Phi_{\psi^{\prime}}\left|\frac{\alpha_{\psi^{\prime}}}{\Phi_{\psi^{\prime}}}\right|^{1 / 2}\right)\right|^{-1}$.

As an example, let us assume that each simulation is of the entire survey, so that we can the calibrate a bias with an error $\sigma_{\psi}$. To reach the required calibration error, $\widehat{\sigma}_{\psi}$, we need $\sigma_{\psi}^{2} / \widehat{\sigma}_{\psi}^{2}$ simulations per bias parameter, and the total number of simulations is $N_{\mathrm{S}}=\sum_{\psi} \sigma_{\psi}^{2} / \widehat{\sigma}_{\psi}^{2}$. Hence, $\alpha_{\psi}=\sigma_{\psi}^{2}$. For a single dark energy parameter, $w=w_{0}$, a single cosmological parameter, $\theta$, and summing over all bias parameters, the fractional change in the DEFoM is

$\Delta \ln \mathrm{F}_{\mathrm{oM}}^{\mathrm{DE}}=-\sum_{\psi}\left|\Delta \mu_{w \psi}\right|^{2} \frac{\widehat{\sigma}_{\psi}^{2}}{\sigma_{\psi}^{2}}$,

so that $\Phi_{\psi}=\left|\Delta \mu_{w \psi}\right|^{2} / \sigma_{\psi}^{2}$. The number of simulations needed for calibration is then

$N_{\mathrm{S}}=\frac{1}{v}\left(\sum_{\psi}\left|\Delta \mu_{w \psi}\right|\right)^{2}$.

If the correlation between the bias and $w$ is zero, no simulations are required. When the correlation between the bias and $w$ is of order unity and the number of bias parameters is $N_{\text {bias }}$, then the number of simulations we require is of order $N_{\mathrm{S}} \approx N_{\text {bias }}^{2} / \nu$. For the simple example of the six bias normalization parameters used in this paper, $N_{\text {bias }}=6$, and for $v=0.1$, we expect $N_{\mathrm{S}} \approx 360$. With more detailed numerical studies using our Fisher Matrix formalism, and the spike, noise, and sinc functional forms, we find $N_{\mathrm{S}} \approx 100$. Given the quadratic scaling with the number of bias parameters, we can expect 
this number to rise rapidly. If we have 100 calibration parameters to measure we may need $N_{\mathrm{S}} \approx 10^{6}$ simulations. However, these simulations may have the same underling simulation, with the effect of each systematic added on.

The resulting variance on the measured calibration is

$\widehat{\sigma}_{\psi}^{2}=\frac{v \sigma_{\psi}^{2}}{\left|\Delta \mu_{w \psi}\right|}\left(\sum_{\psi^{\prime}}\left|\Delta \mu_{w \psi^{\prime}}\right|\right)^{-1}$,

so that the calibration error is inversely proportional to the marginalized Fisher correlation coefficient, $\Delta \mu_{w \psi}$. Again, if this is of order unity the external calibration error is $\widehat{\sigma}_{\psi} \approx \sigma_{\psi} \sqrt{\nu / N_{\text {bias }}}$.

\section{SUMMARY AND CONCLUSIONS}

In this paper we have extended the analysis of cosmic shear to include the effect of spatially varying image distortions on the sky. We have investigated the effect of calibration of biases from external data or simulations, and internally from the same survey on cosmological parameter estimation and the DEFoM, and shown how to minimize the size of the calibration set for a given impact. Spatially varying image distortions convolve the shear signal in Fourier space, and the shear power spectrum, mixing evenparity convergence and odd-parity $\beta$-mode signals. We have found analytic solutions for the biased shear-convergence and $\beta$-mode power from convolution with spike, noise, and sinc-like bias power spectra, which can be generated in realistic cosmic shear surveys. In all cases we have studied, the bias power is equally distributed between convergence and $\beta$-mode power. We find that a scale-dependent multiplicative bias power spectrum model, which has commonly been used in previous studies, is not an accurate approximation.

Convolution and additive biases can be removed from the signal by calibration to external data, or from simulations of the effect of the bias, or by allowing the bias to be fit simultaneously to the data. In such a scenario the absolute value of the bias is unimportant since it will be removed and marginalized over, but the uncertainty in the calibration will propagate into the measurement of cosmological and dark energy parameters. We have carried out an analysis to show how removal and marginalization of the bias, using calibration data and self-calibration from the data itself, will propagate into cosmological parameter estimation and then into the DEFoM. We have applied this to archetypal functions forms for the bias power, spike, noise, and sinc-like functions, and show how each individually, and in combination, affect the DEFoM. We find that calibration and removal of the noise-like bias functions, which affects the largest range of scales, has the greatest effect on the DEFoM, followed by the sinc-like function, which contains an oscillatory cut-off at small scales, while the spike bias has the least effect, covering the smallest range of scales. Overall, a calibration approach appears to require less stringent constraints on bias errors than the algorithmic corrections of the bias. Our results are of relevance to future surveys such as Euclid and LSST, but also to current surveys which use a calibration approach to remove biases.

We have also carried out an optimization of the required calibration error, in order to minimize the number of simulations needed to measure the calibration for a fixed deterioration of the DEFoM. This calculation could also be used to minimize the external data required for calibration.

Finally, our method is general enough that we can extend the formalism to allow the study of bias, bias-removal and the effect of calibration error in the non-linear matter power spectra, baryonic effects on the matter power spectra, photometric redshift calibration, intrinsic alignment calibration, and indeed any effect in the measurement which can be corrected for by calibration. As the formalism is a Pseudo-Cls approach it can account for the effects of the survey window function on the shear power spectrum. This enables the investigation of the effect removing these biases on dark energy and modified gravity experiments.

\section{ACKNOWLEDGEMENTS}

ANT thanks the Royal Society for the support of a Wolfson Research Merit Award while TDK is supported by a Royal Society University Research Fellowship. ANT also acknowledges the support of the UK Space Agency and an STFC Consolidated Grant. We thank Mark Cropper and Tim Schrabback for useful discussions.

\section{REFERENCES}

Albrecht A. et al., 2006, Dark Energy Task Force Report (arXiv:astro-ph/0609591)

Amara A., Réfrégier A., 2008, MNRAS, 391, 228

Amendola L. et al., 2013, Living Rev. Relativ., 16, 6

Bernstein G., 2006, ApJ, 637, 598

Bernstein G., Huterer D., 2010, MNRAS, 401, 1399

Brown M. L., Castro P. G., Taylor A. N., 2005, MNRAS, 360, 1262

Cardone V. F., Martinelli M., Calabrese E., Galli S., Huang Z., Maoli R., Melchiorri A., Scaramella R., 2014, MNRAS, 439 202

Castro P. G., Heavens A. F., Kitching T. D., 2005, Phys. Rev. D, 72, 3516

Cropper M. et al., 2013, MNRAS, 431, 3103

Fenech Conti I., Herbonnet R., Hoekstra H., Merten J., Miller L., Viola M., 2017, MNRAS, 467, 1627

Heymans C. et al., 2006, MNRAS, 368, 1323

Heymans C. et al., 2012, MNRAS, 427, 146

Hildebrandt H. et al., 2017, MNRAS, 465, 1454

Hivon E., Górski K. M., Netterfield C. B., Crill B. P., Prunet S., Hansen F. 2002, ApJ, 567, 2

Huterer D., Takada M., Bernstein G., Jain B., 2006, MNRAS, 366, 101

Ishak M., 2005, MNRAS, 363, 469

Jarvis M. et al., 2016, MNRAS, 460, 2245

Kaiser N., Squires G., Broadhurst T., 1995, ApJ, 449, 460

Kilbinger M. et al., 2013, MNRAS, 430, 2200

Kitching T. D., Taylor A. N., Heavens A. F., 2008, MNRAS, 389, 173

Kitching T. D., Amara A., Abdalla F. B., Joachimi B., Refregier A., 2009, MNRAS, 399, 2107

Kitching T. D. et al., 2012, MNRAS, 423, 3163

Kitching T. D., Taylor A. N., Hood R. K. E., Cropper M., Niemi S., 2016, MNRAS, 455, 3319

Knox L., Song Y.-S., Tyson A. J., 2006, Phys. Rev. D, 74, 023512

Kuijken K. et al., 2015, MNRAS, 454, 3500

Laureijs R. et al., 2011, preprint (arXiv:1110.3193)

Mandelbaum R. et al., 2018, PASJ, 70, S25

Massey R. et al., 2007, MNRAS, 376, 13

Massey R. et al., 2013, MNRAS, 429, 661

Paulin-Henriksson S., Amara A., Voigt L., Réfrégier A., Bridle S., 2008 , A\&A, 484, 67

Planck Collaboration XIII, 2016, A\&A, 594, A13

Taylor A. N., Kitching T. D., 2010, MNRAS, 408, 865

Taylor A. N., Kitching T. D., Bacon D. J., Heavens A. F., 2007, MNRAS, 374, 1377

Tegmark M., Taylor A. N., Heavens A. F., 1997, MNRAS, ApJ, 480, 22

van Uitert E. et al., 2018, MNRAS, 476, 4662 


\section{APPENDIX A: FOURIER MODES OF BIASED SHEAR $\kappa$ AND $\beta$ FIELDS}

The change in the Fourier modes of the convergence, $\Delta \kappa=\widehat{\kappa}-\kappa$, and $\beta$ modes $\Delta \beta=\widehat{\beta}-\beta$, transformed from the measured shear in equation (2), with a spatially varying spin-2 multiplicative distortion of the shear signal, which we write as $m=m_{1}+i m_{2}$, is

$\Delta \kappa(\ell)=\int \frac{\mathrm{d}^{2} \ell^{\prime}}{(2 \pi)^{2}}\left(m_{1}\left(\ell-\ell^{\prime}\right)\left[\kappa\left(\ell^{\prime}\right) \cos 2 \varphi_{\ell \ell^{\prime}}-\beta\left(\ell^{\prime}\right) \sin 2 \varphi_{\ell \ell^{\prime}}\right]-m_{2}\left(\ell-\ell^{\prime}\right)\left[\beta\left(\ell^{\prime}\right) \cos 2 \varphi_{\ell \ell^{\prime}}+\kappa\left(\ell^{\prime}\right) \sin 2 \varphi_{\ell \ell^{\prime}}\right]\right)+c_{\kappa}(\ell)$,

$\Delta \beta(\boldsymbol{\ell})=\int \frac{\mathrm{d}^{2} \ell^{\prime}}{(2 \pi)^{2}}\left(m_{1}\left(\boldsymbol{\ell}-\boldsymbol{\ell}^{\prime}\right)\left[\beta\left(\boldsymbol{\ell}^{\prime}\right) \cos 2 \varphi_{\ell \ell^{\prime}}+\kappa\left(\boldsymbol{\ell}^{\prime}\right) \sin 2 \varphi_{\ell \ell^{\prime}}\right]+m_{2}\left(\boldsymbol{\ell}-\boldsymbol{\ell}^{\prime}\right)\left[\kappa\left(\boldsymbol{\ell}^{\prime}\right) \cos 2 \varphi_{\ell \ell^{\prime}}-\beta\left(\boldsymbol{\ell}^{\prime}\right) \sin 2 \varphi_{\ell \ell^{\prime}}\right]\right)+c_{\beta}(\boldsymbol{\ell})$.

The first terms are the same as for a scalar multiplicative distortion, when the phase angle is $\varphi_{\mathrm{m}}=0$. The second term in the outer brackets of the integrand is due to the spin-2 local phase change of the multiplicative bias mixing in the orthogonal component of the bias.

\section{APPENDIX B: CORRELATIONS OF THE OBSERVED FOURIER MODES}

The correlation of the Fourier modes of the measured convergence field, assuming no intrinsic $\beta$ modes, $C_{\ell}^{\beta \beta}=0$, and for a scale-dependent, scalar multiplicative bias, $m_{0}(\ell)$ where each bias is broken down into spatially constant, deterministic spatial variations and random variations, $m_{0}(\ell)=b_{\mathrm{m}}(\ell)+\delta m(\ell)$ where $b_{\mathrm{m}}(\boldsymbol{\ell})=b_{\mathrm{m}} \delta_{\mathrm{D}}(\boldsymbol{\ell})+\Delta m(\boldsymbol{\ell})$, is given by

$$
\begin{aligned}
\left\langle\widehat{\kappa}(\boldsymbol{\ell}) \widehat{\kappa}^{*}\left(\boldsymbol{\ell}^{\prime}\right)\right\rangle= & (2 \pi)^{2} C_{\ell}^{\kappa \kappa} \delta_{\mathrm{D}}\left(\boldsymbol{\ell}-\boldsymbol{\ell}^{\prime}\right)+\left(b_{\mathrm{m}}\left(\boldsymbol{\ell}-\boldsymbol{\ell}^{\prime}\right) C^{\kappa \kappa}(\ell)+b_{\mathrm{m}}\left(\boldsymbol{\ell}^{\prime}-\boldsymbol{\ell}\right) C^{\kappa \kappa}\left(\ell^{\prime}\right)\right) \cos 2 \varphi_{\ell \ell^{\prime}}+b_{c_{\kappa}}(\boldsymbol{\ell}) b_{c_{\kappa}}^{*}\left(\boldsymbol{\ell}^{\prime}\right)+(2 \pi)^{2} \mathcal{C}_{c_{\kappa}}(\ell) \delta_{\mathrm{D}}\left(\boldsymbol{\ell}-\boldsymbol{\ell}^{\prime}\right) \\
& +\int \frac{\mathrm{d}^{2} \ell^{\prime \prime}}{(2 \pi)^{2}}\left(b_{\mathrm{m}}\left(\boldsymbol{\ell}-\boldsymbol{\ell}^{\prime \prime}\right) b_{\mathrm{m}}^{*}\left(\boldsymbol{\ell}^{\prime}-\boldsymbol{\ell}^{\prime \prime}\right)+(2 \pi)^{2} \mathcal{C}_{\mathrm{m}}\left(\boldsymbol{\ell}-\boldsymbol{\ell}^{\prime \prime}\right) \delta_{\mathrm{D}}\left(\boldsymbol{\ell}-\boldsymbol{\ell}^{\prime}\right)\right) C^{\kappa \kappa}\left(\ell^{\prime \prime}\right) \cos 2 \varphi_{\ell \ell^{\prime \prime}} \cos 2 \varphi_{\ell^{\prime} \ell^{\prime \prime}},
\end{aligned}
$$

$$
\begin{aligned}
\left\langle\widehat{\beta}(\boldsymbol{\ell}) \widehat{\beta}^{*}\left(\boldsymbol{\ell}^{\prime}\right)\right\rangle= & b_{c_{\beta}}(\boldsymbol{\ell}) b_{c_{\beta}}^{*}\left(\boldsymbol{\ell}^{\prime}\right)+(2 \pi)^{2} \mathcal{C}_{c_{\beta}}(\ell) \delta_{\mathrm{D}}\left(\boldsymbol{\ell}-\boldsymbol{\ell}^{\prime}\right) \\
& +\int \frac{\mathrm{d}^{2} \ell^{\prime \prime}}{(2 \pi)^{2}}\left(b_{\mathrm{m}}\left(\boldsymbol{\ell}-\boldsymbol{\ell}^{\prime \prime}\right) b_{\mathrm{m}}^{*}\left(\boldsymbol{\ell}^{\prime}-\boldsymbol{\ell}^{\prime \prime}\right)+(2 \pi)^{2} \mathcal{C}_{\mathrm{m}}\left(\boldsymbol{\ell}-\boldsymbol{\ell}^{\prime \prime}\right) \delta_{\mathrm{D}}\left(\boldsymbol{\ell}-\boldsymbol{\ell}^{\prime}\right)\right) C^{\kappa \kappa}\left(\ell^{\prime \prime}\right) \sin 2 \varphi_{\ell \ell^{\prime \prime}} \sin 2 \varphi_{\ell^{\prime} \ell^{\prime \prime}},
\end{aligned}
$$

and

$$
\begin{aligned}
\left\langle\widehat{\kappa}(\ell) \widehat{\beta}^{*}\left(\ell^{\prime}\right)\right\rangle= & b_{\mathrm{m}}\left(\boldsymbol{\ell}-\boldsymbol{\ell}^{\prime}\right) C^{\kappa \kappa}(\ell) \sin 2 \varphi_{\ell \ell^{\prime}}+b_{c_{\kappa}}(\ell) b_{c_{\beta}}^{*}\left(\boldsymbol{\ell}^{\prime}\right) \\
& +\int \frac{\mathrm{d}^{2} \ell^{\prime \prime}}{(2 \pi)^{2}}\left(b_{\mathrm{m}}\left(\boldsymbol{\ell}-\boldsymbol{\ell}^{\prime \prime}\right) b_{\mathrm{m}}^{*}\left(\boldsymbol{\ell}^{\prime}-\boldsymbol{\ell}^{\prime \prime}\right)+(2 \pi)^{2} \mathcal{C}_{\mathrm{m}}\left(\boldsymbol{\ell}-\boldsymbol{\ell}^{\prime \prime}\right) \delta_{\mathrm{D}}\left(\boldsymbol{\ell}-\boldsymbol{\ell}^{\prime}\right)\right) C^{\kappa \kappa}\left(\ell^{\prime \prime}\right) \cos 2 \varphi_{\ell \ell^{\prime \prime}} \sin 2 \varphi_{\ell^{\prime} \ell^{\prime \prime}}
\end{aligned}
$$

From these expressions we can take $\ell=\ell^{\prime}$, and use the finite-field approximation $\delta_{\mathrm{D}}(\mathbf{0})=A /(2 \pi)^{2}$, to yield the convergence, given by equations (8) and (9) and the $\beta$-mode power given in Appendix $\mathrm{C}$.

\section{APPENDIX C: $\beta$-MODE POWER SPECTRA}

The measured $\beta$-mode power spectrum is

$\widehat{C}^{\beta \beta}(\ell)=\frac{1}{A}\left\langle|\widehat{\beta}(\ell)|^{2}\right\rangle=\int \frac{\mathrm{d}^{2} \ell^{\prime}}{(2 \pi)^{2}}\left(\frac{1}{A}\left|b_{\mathrm{m}}\left(\ell-\ell^{\prime}\right)\right|^{2}+\mathcal{C}_{\mathrm{m}}\left(\ell-\ell^{\prime}\right)\right) C^{\kappa \kappa}\left(\ell^{\prime}\right) \sin ^{2} 2 \varphi_{\ell \ell^{\prime}}+\mathcal{A}^{\beta \beta}(\ell)$,

where

$\mathcal{A}^{\beta \beta}(\ell)=\frac{1}{A}\left|b_{c_{\beta}}(\ell)\right|^{2}+\mathcal{C}_{c_{\beta}}(\ell)$.

If $b_{\mathrm{m}}$ is a constant the bias factors drop out and the measured $\beta$ power becomes

$\widehat{C}^{\beta \beta}(\ell)=\int \frac{\mathrm{d}^{2} \ell^{\prime}}{(2 \pi)^{2}} \mathcal{C}_{\mathrm{m}}\left(\ell-\ell^{\prime}\right) C^{\kappa \kappa}\left(\ell^{\prime}\right) \sin ^{2} 2 \varphi_{\ell \ell^{\prime}}+\mathcal{A}^{\beta \beta}(\ell)$.

A constant noise-power bias, $\mathcal{C}_{\mathrm{m}}(\ell)=\mathcal{C}_{\mathrm{N}}$, will lead to a $\beta$-mode power of $\widehat{C}^{\beta \beta}(\ell)=\mathcal{C}_{\mathrm{N}} \sigma_{\kappa}^{2} / 2$. If we assume a slowly varying scale-dependent shear power spectrum reduces to

$\widehat{C}^{\beta \beta}(\ell)=\frac{1}{2} \sigma_{\mathrm{m}}^{2} C^{\kappa \kappa}(\ell)+\mathcal{A}^{\beta \beta}(\ell)$.

Hence, we see that the mixing of Fourier modes is required to transform from convergence to $\beta$-mode power, and that the assumption of a slowly varying shear power spectrum evenly distributes the measured multiplicative power between the convergence and $\beta$ modes.

This paper has been typeset from a $\mathrm{T}_{\mathrm{E}} \mathrm{X} / \mathrm{L} \mathrm{T} \mathrm{E} \mathrm{X}$ file prepared by the author. 